\title{
VORWORT ZUR ORIGINALAUSGABE
}

Im Jahre 1964 haben M. L. ZETuIN und ich zum erstenmal die Idee der Herausgabe dieses Buches beraten. Im Winter 1964/65 schrieben wir einen Utbersichtsvortrag „Kollektive von Automaten und Verhaltensmodelle“ für die III. Allunionskonferenz zu Fragen der automatischen Steuerung und im Winter 1965/66 den Vortrag ,Automata and models of collective behaviour" für den III. IFAC-Kongre $B$ in London. Beide Vorträge betrachteten wir als ersten Inhaltsentwurf des Buches. Auf der Grundlage der beiden Vorträge wurden zwei weitere Utbersichtsvorträge gehalten, und zwar von M. L. Zetwn in Prag und vom Autor dieses Buches in Edinburgh. Im Sommer 1966 wollten wir mit der Vorbereitungsarbeit für das Buch beginnen, jedoch machte der tragische Tod M. L. ZeturNs diese Pläne zunichte.

Im Jahre 1969 wurde postum ein Sammelband von Arbeiten M. L. Zeturns mit dem Titel „Untersuchungen zur Automatentheorie und der Modellierung biologischer Systeme" herausgegeben. Im Sammelband wurde unter die bis 1966 angeführten Arbeiten über das kollektive Verhalten von Automaten ein Schlußstrich gezogen, und im Anhang wurde ein kurzer Utberblick über eine Reihe späterer Resultate gegeben. Der Sam melband umfaßte den ursprünglichen von uns 1964 beratenen Plan des Buches. Jedoch schon zum Erscheinungszeitpunkt des Buches von M. L. ZETurN hatte man eine Reihe neuer Ergebnisse erhalten, die in einigen Fällen wesentliche Bedeutung für die Thematik hatten.

Eine Vielzahl von Diskussionen überzeugte den Autor davon, daß die Idee der Herausgabe des Buches aktuell blieb. Um die Geschlossenheit der Darstellung zu bewahren, wurde im Buch die Darlegung einiger Fragen des Sammelbandes von M. L. ZETLIN wiederholt. Außerdem wurden Ergebnisse einer Reihe von Zeitschriftenartikeln verwendet, deren Autoren in der Regel Teilnehmer des jährlichen Seminars des Kybernetiklaboratoriums der Leningrader Abteilung des zentralen mathematisch-ökonomischen Instituts der AdW der UdSSR sind. In den Anmerkungen zum Buch sind die Autoren der dargelegten Ergebnisse angeführt, was natürlich nicht die Utbertragung der Verantwortung für die Qualität des Buches auf die erwähnten Personen bedeutet.

Die Auswahl des Materials für das Buch wurde durch folgende Überlegungen bestimmt: erstens durch das eigene wissenschaftliche Interesse des Autors und das wissenschaftliche Interesse derjenigen Personen, mit denen der Autor langjährige wissenschaftliche Kontakte unterhält; zweitens durch das Streben danach, ein häufig äußerst verschiedenartiges Material, das nur durch den einheitlichen methodologischen Zugang verknüpft war, einheitlich und zusammenhängend darzustellen; drittens durch den Wunsch, einen genügend großen Kreis inhaltsreicher Fragestellungen zu erfassen, um die Möglichkeit zu demonstrieren, in der Sprache des kollektiven Verhaltens von Automaten über komplizierte Objekte verschiedenartiger Natur zu sprechen.

Ein Teil des Buchmaterials erscheint im Kleindruck. Ein Leser, der sich im wesent- 
lichen nur für die inhaltliche Seite einer Frage interessiert, kann diese Stellen ohne Schaden für das Verständnis übergehen.

Der Autor drückt seine aufrichtige Dankbarkeit all jenen aus, die ihm freundlicherweise die Verwendung ihrer Resultate in diesem Buch ermöglicht haben und bittet alle Personen, deren interessante Resultate auf dem Gebiet der Untersuchung von Modellen des Verhaltens von Automaten in zufälligen Medien und von Modellen des kollektiven Verhaltens in diesem Buche nicht erwähnt werden, um aufrichtige Entschuldigung. Aber leider kann man das nicht umfassen, was unumfaßbar ist.

Die ständigen Kontakte mit M. M. Bongard, W. A. Borowikow, E. M. BraWermaN, W. S. Gurfinkel, S. M. Meerkow, L. I. Rosonoer, I. I. PJatezki-Schapiro und die Diskussion sowohl eigener wissenschaftlicher Ergebnisse des Autors als auch dieses Buches brachten einen, sehr großen Nutzen. Der Autor zweifelt daran, da B er die Arbeit ohne die ständige Hilfe von W. B. Marachowski, W. A. Pestschanski und L. JA. RosenblJum und deren unermüdlichem Bemühen, Fehler im Manuskript festzustellen, zu Ende geführt hätte. Der Autor bedauert es, wenn diese sich fast in ein sportliches Spiel verwandelte Beschäftigung der Fehlersuche nicht zu Ende geführt wurde. Seine besondere Dankbarkeit drückt der Autor dem Redakteur des Buches, D. A. Pos. PELow aus, der viel Arbeit aufgewendet hat, um das Buch gut lesbar zu machen.

Leningrad, Januar 1972

Autor

\section{VORWORT DES HERAUSGEBERS}

Die reale Welt zu erkennen, erfolgt im allgemeinen auf dem Wege, abstrakte Modelle für Objekte oder Erscheinungen zu schaffen, diese Modelle zu untersuchen und zu verbessern und aus der Erforschung der Modelle Schlüsse auf das modellierte Objekt zu ziehen. Die stürmische Entwicklung technischer Wissenschaften hat in den letzten Jahrzehnten zu realen, großen Systemen geführt, deren Verhalten zu beschreiben zwar für die Praxis ungemein wichtig ist, aber wegen der zufälligen Einflüsse und der zwischen den Systemteilen existierenden Wechselbeziehungen große Schwierigkeiten bereitet.

Es gehört deshalb seit langem zu den reizvollen Arbeiten, mit den durch die Mathematik zur Verfügung gestellten Methoden und mit ihrem Formalismus Systeme oder Systemteile zu beschreiben. Entsprechende Arbeiten sind Teil mathematischer, praxisorientierter Forschung, wenn auch häufig die Restriktionen, die bei der Modellierung heute noch erforderlich sind, diese Praxisbezogenheit verschütten. Da dergleichen Forschungsarbeiten aber weltweit betrieben werden, ist es zu begrüßen, da $B$ der Akademie-Verlag in sein Veröffentlichungsprogramm eine neuere, sowjetische Arbeit aufgenommen hat.

Ich möchte für das Lesen des deutschsprachigen Manuskriptes, für zahlreiche Diskussionen, für Hinweise und Vorschläge für die Endfassung Herrn Prof. Dr. Helmut ThIELE, Humboldt-Universität zu Berlin, herzlich danken. Ferner geht mein Dank an den Autor für den Kontakt während der Utbersetzung und der Vorbereitung der Herausgabe. 